\title{
Contradicciones en la educación inclusiva a través de un estudio multiescalar
}

\author{
Begoña Vigo Arrazola, Belén Dieste Gracia \\ Universidad de Zaragoza
}

\section{RESUMEN}

En un contexto en el que los servicios de educación han sido transformados en una dirección neoliberal, tanto la inclusión como la participación de las familias son aspectos destacados en una educación de calidad. Sin embargo, los efectos del Nuevo Mercado, en términos de participación, justicia y equidad, no han sido favorables para la elección de escuelas en áreas geográficas con desventaja social y económica. Este artículo intenta mostrar la comprensión que tienen estas escuelas como espacios educativos a partir del análisis en profundidad de una escuela. El estudio realizado desde un proyecto etnográfico multiescalar en ocho escuelas de Aragón, en España, a través de observación participante y entrevistas a maestros/as, alumnos, padres, madres, equipos psicopedagógicos e inspectores, muestra cómo los miembros de esta escuela se presentan a sí mismos de distinta manera a cómo lo hacen la administración, los medios de comunicación o los miembros de la misma ciudad. El conocimiento del lugar y espacio, la conexión de la vida de los alumnos con el curriculum y el desarrollo profesional de los profesores son estrategias que dan cuenta de las prácticas de educación inclusiva en la escuela. El artículo señala los diferentes discursos como consideraciones para la administración educativa.

Palabras Clave: etnografía, escuela estigmatizadas, participación de familias, inclusión.

\section{Contradictions in inclusive education through a multi-scalar study}

\section{ABSTRACT}

In a context where education services have been transformed in a neo-liberal direction, the inclusion and the participation of parents in schools is stressed as an important aspect of quality. Nevertheless, the effects of the new market, in terms of participation, justice and equity are negative on school availability and selection in disadvantage areas. This article tries to show the understandings of these schools as educational spaces, through a deep analysis of one school. The study carried out from an ethnographic research multi-scalar project in eight schools of Aragon in Spain from participant observation and interviews with teachers, students, parents, counsellors and inspectors, shows how members of schools are present as in a different way to representations of the administration, media or members of the town. Results show as the knowledge of spatial conditions, connection between students' life and curriculum and professional development, are strategies closer to an inclusive school. This article highlights different contradictions to consider by the educational administration.

Keywords: ethnography, school stigmatisation, parental participation, inclusion.

En un contexto global donde el mercado educativo ha contribuido a destacar los resultados como índice de calidad de las escuelas, se hace hincapié en la descentralización, la comercialización y la responsabilidad individual (Beach, 2010). La influencia del sector privado en la elección de la escuela, la competencia y la participación de los padres, como han señalado diferentes investigaciones (Ball, 1994; Beach, Dovemark, Schwartz y Öhrn, 2013; Gewirtz, Ball y Bowe, 1995; Apple, 1996) han tenido importantes implicaciones en el incremento de las condiciones de exclusión frente a la inclusión.

Actualmente, en la mayoría de los países europeos, el Estado se ha transformado radicalmente en una dirección neoliberal, promoviendo cambios significativos en relación con el trabajo docente. Una educación inclusiva parece el lema que debe guiar la práctica en la escuela. Conferencias e informes internacionales subrayan la inclusión como referente para conseguir una educación de calidad que sea más justa y equitativa (Engelbrecht, 2013). Los profesores deben actuar de nuevas maneras y formar nuevas alianzas con las familias y la comunidad para mejorar la calidad del aprendizaje. Diferentes trabajos subrayan la importancia de la participación de las familias en un sistema educativo de calidad (Bolívar, 2006; Garreta, 2014; San Fabián, 1994, Smith, 2006) en una escuela inclusiva (Ainscow, Booth y Dyson, 2006; Vigo, Dieste y Julve, 2016). En este sentido, ha habido un cam- 
bio en la ideología (Beach, 2010; Goodson y Lindblad, 2011); las escuelas deben desarrollarse según las demandas y necesidades sociales.

La investigación muestra como las prácticas educativas que conectan la escuela y la vida o que establecen vínculos con las familias y la comunidad contribuyen al desarrollo de las escuelas para facilitar el aprendizaje de todos y todas (Arnaiz, 2003; López Melero, 2012; Susinos y Parrilla, 2008; Vigo y Soriano, 2014, 2015; Vigo, et al., 2016a). Cuando los valores de la escuela y los de la familia coinciden, se desarrolla un sentimiento de pertenencia al centro (Crozier y Davies, 2007). Sin embargo, cuando los valores de las familias no coinciden con los de la escuela, éstas se pueden encontrar más condicionadas para conectar y participar (Bergnehr, 2015; Lareau, 2000; Schecter y Sherry, 2009; Therodorou, 2008). Las prácticas de reflexión y análisis sobre el funcionamiento de la escuela que llevan a cabo el profesorado y la comunidad se constituyen como una base de referencia para el desarrollo profesional y para el desarrollo de la participación en una escuela que pretende ser inclusiva (Ainscow, et al., 2006; Vigo, Dieste y Thurston, 2016).

La normativa nacional e internacional sobre educación subraya entre sus bases una educación inclusiva (UNESCO, 2008). En los distintos países, se desarrollan decretos de inclusión que presentan una abstracción y aproximación a la 'educación inclusiva'. Desde una perspectiva centrada en una escuela que se centra en las necesidades de alumnos individuales y medidas excepcionales (Martínez, 2011), los sistemas educativos construyen los discursos en torno a la inclusión en educación. No obstante, como aspiración a una educación democrática (Escudero y Martínez, 2012), el proyecto de inclusión de una escuela debería considerar las experiencias de todos sus estudiantes (Slee, 2012). Esta cuestión es problemática en un contexto que promueve la diferenciación de grupos de población por sexo, capacidades o etnias. Más específicamente, en países en los que el nivel de inmigración ha aumentado significativamente, se han llegado a reforzar, en ocasiones, situaciones de segregación en contextos educativos (Essomba, 2012).

En España, en las últimas décadas ha habido un rápido aumento de la inmigración. Esto, junto con las características de la elección de escuela, ha generado nuevas cuestiones en los debates sobre oportunidades educativas y desigualdades en espacios estigmatizados en áreas económicamente pobres (Bernal, 2005; Escardíbul and Villarroya, 2009; Olmedo, 2008). Las preguntas surgen en relación con cómo la elección de centro puede aumentar o disminuir la desventaja de los diferentes grupos con respecto a la población mayoritaria (Carrasco, Pamiés y Bertran, 2009; Jackson, Jhonsson y Rudolphi, 2012; Slee, 2012). Esta cuestión conlleva un importante reto en todos los países que pretenden ofrecer a sus estudiantes una educación igualitaria. Según Beach (2017) es un problema de equidad y justicia vinculado a la política educativa y a la representación de intereses, prácticas y liderazgo de organizaciones educativas en un contexto internacional más amplio.

Este artículo se centra en las situaciones de segregación y participación en contextos educativos y, de forma más concreta, en la participación de la familia en los procesos escolares de ocho escuelas de Aragón. Más específicamente, se profundiza en el análisis detallado de una escuela en la que los niveles de desempleo y migración de la población son elevados.

El índice de abandono escolar temprano en España es del 20\% y en Aragón del 19,5\% en el curso académico 2014/2015. Sin embargo, parece que hay enormes diferencias entre los contextos geográficos, los barrios y las escuelas. En áreas, donde se encuentra más del 50 por ciento de los residentes viviendo con apoyo económico, existe una situación en la que más de la mitad de las familias que viven por debajo de la línea de pobreza (Flores, Gomez y Renes, 2016). En este contexto, proporcionar a los estudiantes unas condiciones de educación igualitaria es un reto (Beach y Sernhede, 2012; Moreno y Bruquetas, 2011; Gil, de Pablos y Martínez, 2010; Chusseau y Hellier, 2011). Estudios en diferentes países muestran que los resultados escolares parecen considerablemente más bajos en barrios desfavorecidos y el abandono es mayor (Flores et al., 2016). La investigación hace pensar que los estudiantes no obtienen una educación que se adapte a sus necesidades y experiencias (Calderón, 2014; Carrasco et al., 2014; Öhrn, 2012). La segregación escolar significa no sólo que los niños de diferentes orígenes raciales y étnicos asistan a diferentes escuelas, sino también que en estas escuelas los niños empiezan a pensar de manera diferente sobre el mundo y su lugar en él (Carrasco, et al., 2016; Logan, Mincar y Adar, 2012). Así, comienza una separación tanto mental como física en estos contextos (Öhrn, 2012; Bernal, 2005; Jackson et al, 2012).

Como señalan distintas investigaciones internacionales, la inadecuación parece obvia cuando se describen y analizan las prácticas y las experiencias de vida que tienen lugar en la realidad educativa. Sin embargo, es posible ver cómo las escuelas, superan y salen adelante en un contexto que lejos de reconocer sus potencialidades subraya su situación de desventaja (Beach, et al., 2013). En España, cuando se revisan las investigaciones existentes en relación con la participación de alumnos y familias es posible observar la escasa referencia a una línea metodológica de investigación que profundice en el procedimiento, y que muestre cómo se lleva a cabo esa participación.

\section{El contexto de la investigación}

La investigación, en la que se basa este trabajo, aborda las prácticas y los discursos sobre las experiencias de ocho escuelas de Aragón (España). Tres de ellos comparten las características de ser espacios desfavorecidos. Este artículo especifica el análisis de una escuela ubicada en el área periurbana de una ciudad.

La escuela pertenece a un barrio construido en los años sesenta y principios de los setenta, donde se asentaba población obrera, como resultado de la industrialización y del consecuente movimiento de la población del campo a la ciudad. En las últimas décadas, como en barrios similares, ha tenido lugar una paulatina desindustrialización.. La historia de ésta zona de la escuela es importante (Gil, 1966), ya que lo que aparece hoy y ahora tiene históricamente referentes de clase, que a través de los medios de comunicación se proyectan en el presente y el futuro (Beach et al, 2013). Esto significa que es una zona que ha mantenido un estigma del pasado (Flores et al., 2016; Gudmundsson, 2013; Møller, 2013), pero ahora con una población multicultural que sufre la creciente pobreza, el aislamiento social, la estigmatización racial, el desempleo, y el declive en comparación con las poblaciones anteriores (Beach \& Sernhede, 2012). Así se refleja en el 'Observatorio de la Realidad de Nuestro Barrio" o en el proyecto educativo de la escuela. El barrio actualmente, cuenta con una población en torno a los seis mil doscientos treinta y cinco habitantes.

En los últimos años se ha asentado en el barrio un importante núcleo de población inmigrante, fundamentalmente africana (subsahariana y magrebí, que tienen en común el hecho de ser musulmanes), y un porcentaje muy alto de la población de etnia gitana de toda la ciudad. Todo esto se debe al bajo coste de la vivienda que desde siempre ha venido caracterizando a este barrio. 
En cuanto al aspecto laboral, una parte de los habitantes del Barrio se dedican principalmente a trabajos en administraciones públicas, actividades industriales (metal y construcción), de servicios (comercio y hostelería) y talleres de reparaciones. Existe otro núcleo de población numeroso dependiente de ayudas sociales y con niveles culturales muy bajos; suelen estar en el paro o dedicarse a la recogida de diversos productos. Esta población desde hace dos años ha aumentado debido al desalojo de viviendas ocupadas por familias gitanas en el casco antiguo de la ciudad y su posterior traslado a este barrio por el bajo coste de los alquileres.

Estas condiciones han creado lo que Møller (2013) en otro contexto identifica como una "etnificación" de la pobreza y la segregación, que parece estar vinculada a la hegemonía de clases (Calderón, 2014).

\section{Metodología}

Los datos del presente artículo proceden principalmente de un proyecto etnográfico multiescalar (Eisenhart, 2016) llevado a cabo en 8 escuelas ("Familias y escuelas. Discursos y Prácticas cotidianas sobre la participación en la educación obligatoria". EDU2012-32657) entre los años 2012 y 2015. En total han tenido lugar más de 800 horas de observación participante en las escuelas y más de cien entrevistas con la dirección de la escuela, maestros, alumnos, padres, inspectores y trabajadores sociales.

Alrededor del $40 \%$ del tiempo dedicado a la observación participante se realizó en reuniones con los padres, entradas y salidas de la escuela. El 60\% de la observación participante ha tenido lugar en un aula de cada escuela. La escuela que se especifica en este artículo es de Educación Infantil y Primaria con alumnos de 3 a 12 años.

La intención en este artículo es considerar las prácticas de la escuela y los discursos sobre la misma y contrastar los puntos de vista comunes procedentes de la escuela con los discursos negativos externos.

La etnografía, ha permitido ilustrar detalles individuales y complejos. Por esta razón, se han tratado de garantizar las bases teóricas y los principios etnográficos básicos. En este sentido, ha sido importante enfatizar las prácticas activas y creativas llevadas a cabo en las escuelas y su relación dialéctica con las prácticas sociales humanas, la conciencia humana, las estructuras sociales y las condiciones materiales y de investigación. Resultados: Sensibilidad hacia el lugar y el espacio. Entre la representación local y la representación externa

Los resultados de una investigación etnográfica tienen sentido cuando se explican desde una relación dialéctica con otros estudios, realidades analizadas y reflexiones. No son datos aislados. El estudio ha proporcionado ideas importantes para entender los diferentes discursos, internos y externos, acerca de las escuelas. Un resultado específicamente relevante y consistente se refiere a la comprensión y la interpretación de la desigualdad educativa y las medidas diseñadas por las políticas educativas para enfrentarse a ella. Estas políticas parecen ignorar características importantes de la situación social y cultural de la población (Beach et al, 2013; Martínez, 2011), o más específicamente, la relación entre la ubicación socio-espacial, su representación y la formación de identidades diferenciadas (Beach \& Sernhede, 2012; Öhrn, 2012). Así, los profesores de esta escuela se presentan como distintos de otras y las distinciones que ellos señalan no coinciden normalmente con las transmitidas por las representaciones externas.
“Aquí el alumnado es distinto y las familias son distintas. Los profesores también somos distintos. Tenemos una sensibilidad especial. ... nos preocupa muchísimo la convivencia ... Nosotros somos unos trabajadores y la escuela es de las familias /... / Este es un colegio casi permanentemente de puertas abiertas, para que los padres confíen y vean lo que hacemos con sus hijos. (Director)

Esta sensibilidad de los profesores y las estrategias que utilizan para salir adelante parecen estar asociadas con un aspecto fundamental como es el éxito educativo, tal y como señala la investigación internacional (Barnard, 2004; Castro, Expósito, Lizasoain, López y Navarro, 2014; Fan y Chen, 2001; Harris and Goodall, 2008, 2009; Lindsay y Davis, 2009; Vigo, et al.,, 2016a). Estrategias como el conocimiento del lugar y espacio, la conexión de la vida de los alumnos con el curriculum y el desarrollo profesional de los profesores dan cuenta de las prácticas que llevan a cabo en la escuela y de las contradicciones de las políticas de inclusión.

\section{Conocimiento del espacio}

Uno de los puntos observados, de acuerdo con otros estudios, es que aunque las escuelas ubicadas en áreas desfavorecidas se presentan como distintas de las escuelas en otras áreas residenciales, estas distinciones no se corresponden por lo general con las referencias comúnmente utilizadas por la administración o los medios de comunicación (Beach \& Dovemark, 2009). Fuera de la escuela, en la ciudad o desde la propia administración, se destacan al describirla marcadores como la diferencia o las relaciones económicas, la localización social y urbano-geográfica. Desde dentro, los profesores ven otras cuestiones que son más importantes para ellos (Öhrn, 2012). Éstos subrayan su modo de hacer y usan marcadores como la cultura, el lenguaje, la raza, la religión o la etnicidad (Beach \& Beach y Sernhede, 2012).

Nosotros aquí somos la escuela de inmigrantes y gitanos / ... / siempre piensan en nosotros como escuela excluida /.../ Estamos descritos como una escuela diferente (y) esto se utiliza para decir porqué fallamos ... Pero nuestros problemas no tienen que ver con la religión o la etnicidad ... tienen que ver con (cómo) somos vistos por otros

Esa importancia atribuida al déficit como una categoría espacial, urbano-geográfica más que nacional, étnica o racial (Öhrn, 2012), se proyecta dentro de las propias escuelas y a veces parece afectar de manera interactiva. El trabajador social de la escuela señalaba como a "la población mayoritariamente gitana el colegio es algo que les viene grande".

En esta investigación se ve que la escuela parece formar parte de una realidad social compleja que es influenciada interactivamente a través de las representaciones sociales y mediáticas. La escuela es considerada como diferente de otras porque sus residentes recuerdan continuamente que se trata de una realidad desfavorecida debido a la ubicación de su lugar de domicilio y sus características (Beach y Sernhede, 2012; Calderón, 2014; Muñoz, Vázquez y Vázquez, 2003; Öhrn, 2012). La escuela tiene representaciones negativas y es vista como 'problemática' y con necesidad de ayuda o tratamiento especial (Beach \& Sernhede, 2012). Esa visión tiende a reforzar la exclusión. El Director de la escuela aludía "al desconocimiento de la realidad /.../, no desde el cole sino desde fuera. Se producen muchos rumores que no son ciertos". 
Esta escuela es similar a otras escuelas 'sin dificultades' y, al mismo tiempo, muy diferente de ellas. Son similares porque están comprometidos con una buena educación y quieren influir en los alumnos y familias (Beach \& Dovemark, 2009). Las diferencias respecto a otras escuelas se reflejan en las declaraciones sobre lo que esperan de las administraciones educativas y sus representantes (Beach \& Sernhede, 2012; Öhrn, 2012). Como señalaba el director:

La administración tiene más y menos sensibilidad, depende. A veces parece que están más receptivos y hay un poco más de sensibilidad, pero cuando llega el momento de hacer acciones concretas y ejecutar, pues /... / Por ejemplo, ahora mismo, con la escolarización, 40 familias de la ciudad no han podido entrar en los colegios de su barrio. ... La baremación permite que en este barrio con más de 80 niños nacidos para entrar en septiembre solo se hayan matriculado 28.

Estas vivencias y sus significados con frecuencia contrastan con los discursos de una educación inclusiva. Estas son situaciones a las que las escuelas se enfrentan diariamente y tienen que aprender a tratar. A través de sus discursos y acciones, es posible identificar una forma compartida de tratar estos problemas dentro de la escuela. Se puede vislumbrar un compromiso con la acción colectiva (Beach \& Dovemark, 2009; Öhrn, 2012) en relación con los deseos de "hacer" a pesar de las pedagogías fuertemente individualistas de las escuelas. Integrar la vida de los alumnos en la escuela y el desarrollo profesional de los profesores se presentan como estrategias de interés.

\section{Integrando en la escuela la vida de los alumnos}

Cuando se investiga en el centro y en el aula el tipo de prácticas pedagógicas que se llevan a cabo, es posible ver que esta escuela hace un reconocimiento positivo de los problemas que estamos investigando. Los profesores se centran en investigar y descubrir los valores, creencias y costumbres de las familias dentro y fuera de la escuela, y por ende más allá de las fronteras institucionales. Se pudo observar que los maestros estaban particularmente comprometidos al tratar de aprender acerca de las familias y sus valores, situándose dentro del contexto del entorno inmediato de la escuela (véase también Jacobs, 2008; Mills and Gale 2004). La estrategia se podía ver en ejemplos que mostraban cómo los profesores investigaban las vidas y experiencias de las familias a través de actividades curriculares (Bouataz 2007; Vigo y Soriano, 2015). Un ejemplo es cuando una maestra trataba de aprender de las experiencias de las familias en el aula.

Durante dos años he estado haciendo un proyecto sobre los nombres de los estudiantes, ya que es la primera identidad de los estudiantes. Investigan en casa sobre el origen de su nombre, qué significa, por qué se les puso, dónde vivían en ese momento. Así conozco algo de sus orígenes. Esta actividad está dentro de un proyecto sobre familias. (Entrevista maestro)

La vida y la cultura de los alumnos fuera de la escuela también estaban presentes en el aula durante una práctica de "texto libre" vinculada a la enseñanza de la escritura. Los estudiantes proponían temas sobre sus experiencias fuera de la escuela y permitía a los profesores y compañeros obtener más conocimiento y comprensión de sus vidas (Jacobs, 2008).

Estamos haciendo Texto libre. Cada niño-a escribe un texto. /.../ Están concentrados en su escritura. A veces paran de escribir y hacen algún comentario en voz alta. De vez en cuando, levantan la mano y preguntan. /... / Al terminar la clase es posible ver textos cortos y más largos. Francisco, un niño romaní, escribe sobre su padre vendiendo algunos caballos. Nase dice que una jirafa no sabe hablar francés en la escuela. /.../ Las preguntas de los niños y la maestra sirven para desarrollar el contenido del texto, revisar ortografía y puntuación y explicar reglas y normas que van surgiendo. (Notas de campo 23/10/2014)

El valor que los maestros otorgan al entorno familiar también es destacado por un maestro que hablaba sobre la necesidad de conocer a todas las familias de sus estudiantes y entenderlas.

Estoy deseando conocer a las familias personalmente y culturalmente porque de lo contrario no puedo entenderlas y no puedo entender a mis estudiantes. ... Siempre les digo a las familias que hablen de sí mismas y que me digan lo que piensan. (Entrevista maestro)

En otros casos, los profesores realizaban actividades educativas con el objetivo de ampliar la comprensión de la población escolar en todo el centro. Un ejemplo fue cuando las tradiciones culturales de las familias se presentaron a través de la preparación y desarrollo de una mesa redonda titulada "Háblame sobre ti para poder conocerte".

Profesores y familias preparan una mesa redonda. Tras un proceso de discusión plantean diferentes temas. Deciden que una madre guineana hablará de una planta típica de algodón de su país. Otra mamá de Perú hablará de la Navidad en su país, una madre marroquí describirá las bodas en una región de Marruecos y una madre gitana hablará de la educación romaní. Un profesor de español explicará la tradición de la "Tronca" en Aragón y un profesor francés hablará sobre la Pascua en Francia /..../ 60/70 personas son la audiencia, en su mayoría mujeres de origen africano. También hay un número representativo de población gitana y muy pocos habitantes locales y latinoamericanos. También hay unos 5-6 profesores y el equipo directivo. (Notas de campo)

En otra ocasión, una maestra contaba su experiencia fuera de las horas de clases.

El fin de semana pasado fui a una fiesta africana que tuvo lugar cerca de la escuela. Todas sus familias estaban allí. Conocí a tres familias de mi clase y me di cuenta de que lo que se hace en el aula puede ser cambiado. La fiesta es un pequeño punto de referencia que puede servir para mi clase. (Entrevista maestra)

En esta investigación se puede ver cómo los maestros se preocupaban por entender los intereses y preocupaciones de las familias (Schecter y Sherry, 2009). Conocer a las familias era considerado importante y necesario para los maestros. Éstos querían dar valor a la cultura de las familias en el proceso de enseñanza. Reconocían que la escuela responde, generalmente, a valores culturales dominantes, en lugar de prestar atención a los valores culturales individuales de cada estudiante (Beach et al., 2013). Ellos querían cambiar esto escuchando a las familias y aceptando sus culturas. De este modo, la escuela creaba un entorno de apoyo, valoración y legitimación de la experiencia que el niño trae de su hogar. Los valores de los estudiantes fuera de la escuela son reconocidos por la cultura escolar dominante, que los escucha y reconoce (Conteh y Kawashima, 2008). 


\section{Profesores que reflexionan y participan en la investigación}

Los profesores reconocen y refuerzan el valor de las prácticas educativas teniendo en cuenta la vida de sus alumnos fuera de la escuela y teniendo en cuenta a sus familias. De este modo, una dinámica de refuerzo y desarrollo del modelo de acción que habían ido construyendo en interacción coherente con su contexto (Wenger, 2001) guíaba el día a día de su desarrollo profesional.

Durante la investigación llevada a cabo, en los procesos de observación participante, los profesores parecían tomar conciencia de su conocimiento situado (Niemeyer, 2006). Cuando se atendía a las experiencias que llevaba a cabo cada profesor y al conocimiento que incorporaban durante el proceso de la investigación, ellos también eran observadores activos, buscando y ampliando experiencias.

Los profesores, como practicantes reflexivos, se mostraban críticos y disconformes con sus prácticas, buscando el por qué de su propio trabajo y no solo el cómo (Boylan y Woolsey, 2015). Se enfrentaban a su propia práctica preguntándose por qué era importante conocer a las familias, cuando destacaban la necesidad de conocerlas. Una maestra especificaba cómo "las maestras y maestros podemos crear experiencias de aula diferenciadas gracias a que nos movemos, atravesamos, compartimos conocimiento con nuestros contextos, en actos de relación intelectual" (Clavero, 2016, 12).

De forma explícita otra maestra señalaba cómo la investigación había contribuido a una revisión de sus prácticas durante el proceso de la investigación. "Ha modificado mi práctica, me ha servido para repensar lo que estaba haciendo, la forma de hacerlo" (Maestra). Los profesores en interacción con el investigador parecían hacerse conscientes del conocimiento práctico pero también de la necesidad de desarrollar otras prácticas (Hale, Snow-Gerono y Morales, 2008) vinculadas al desarrollo del curriculum. Todo ello tenía lugar a través de las conversaciones reflexivas sobre la práctica o desde el análisis de la interacción en la clase en el día a día.

Como todo proceso de conocimiento, la devolución de la información remitió a procesos de discusión e intercambio de conocimiento a través de un debate colectivo (Cerletti, 2013) acerca de la participación de la familia en la escuela, de la importancia de sus voces en los procesos de enseñanza y aprendizaje en las aulas, y del tiempo que necesitaban para ello. Asimismo, tuvo lugar la reflexión y el cuestionamiento de aquello que les resultaba familiar, poniendo en evidencia su propia práctica (Delamont y Atkinson, 1994). Como señala una maestra: "Te hace pensar en otros posibles referentes /... / nos abre la oportunidad de pensar sobre metodología, agrupaciones, inclusión y prácticas docentes". Esa conceptualización y análisis que se aporta desde el estudio etnográfico pareció servir como elemento de reflexión teórica sobre sus prácticas, de consciencia y de contraste con otros modelos de acción, conectando la experiencia individual y colectiva situada con un proceso de transformación (Niemeyer, 2006).

En mi caso particular, ha supuesto una renovación valiosa cuyo fruto es el encuentro profesional, intelectual y humano. Una forma de hacer escuela a través del placer de aprender, abandonando aquello que supuestamente sabemos para reconstruir realidades educativas.

La idea de desarrollo profesional y el deseo de 'hacer' se reforzaron durante el estudio etnográfico (Woods, 1996). Profesores y familias señalaban el largo camino por recorrer en el proceso, buscando alternativas y opciones de desarrollo en su propio contexto.

\section{Discusión}

Este artículo trata de contribuir a la comprensión de la justicia en educación y en una escuela para todos, donde determinados espacios pueden ser entendidos como algo más que un objeto de déficit y rechazo. Se ha pretendido subrayar qué tipo de prácticas de enseñanza se llevan a cabo y cómo, desde la fundamentación de una investigación etnográfica que proporciona conocimiento contextualizado y detallado, desafiando la investigación y propuestas que van de arriba abajo.

El estudio presenta un espacio de educación inclusiva que se va construyendo atendiendo a las innovaciones y las posibilidades de desarrollo que son significativas para la población en ese espacio. En esta escuela los profesores reconocen que las familias tienen algo que aportar, consideran su identidad y la del entorno. Reconocen y hacen uso de las fortalezas de la población, la cultura y sus antecedentes. De este modo, llevan a cabo prácticas de enseñanza centradas en los estudiantes que permiten participar y aprender a todos.

Los maestros reconocen las contribuciones derivadas de la atención a la cultura familiar para la enseñanza y el aprendizaje, pero también reconocieron una contribución a la satisfacción de las familias y al enriquecimiento comunitario (Theodorou 2008). Señalaron cómo la legitimación de la cultura familiar podría proporcionar una oportunidad importante para construir puentes entre la escuela y las familias. Cuando los maestros mostraron receptividad y apoyaron a las familias, la participación de los padres en la vida escolar aumentó (Epstein 2001, Lewis, Kim y Bey 2011).

Otra característica importante de los resultados es que cuando los maestros y líderes de una escuela tienen un compromiso social, esto puede facilitar los intentos de familiarizarse con las familias y de incorporar ese conocimiento de las mismas para mejorar y enriquecer el proceso de enseñanza. Esto también se vio en el trabajo al que se refirieron anteriormente Bouakaz en Suecia (2007) y Crozier y Davies (2007) en Inglaterra. En la escuela había un compromiso claro con el desarrollo académico de los estudiantes y en todos los casos los profesores trataron de enfatizar la familia con el propósito de mejorar el aprendizaje. Respetar y valorar a los miembros de la comunidad son rasgos que caracterizan las prácticas en esta escuela.

La atención a las culturas de las familias inmigrantes en la escuela podría ser identificada como un aspecto que los maestros consideran en relación con la transformación de las prácticas docentes y la mejora de los resultados de aprendizaje (Bouataz, 2007, Schecter y Sherry, 2009). No obstante, estas estrategias eran reguladas y controladas por los mismos profesores. Esto significa que el conocimiento de la casa / comunidad (fuera) pasa al contexto pedagógico (escuela) para ayudar a que se adapten a nosotros (Beach et al., 2013). Las contradicciones con respecto al uso de estas prácticas pedagógicas que pueden extraerse de detalles etnográficos como éstos parecen evidentes. La voz de la escuela dice que es inclusiva, que es una escuela para todos, y que todos pueden tener éxito. Sin embargo, esto sólo es cierto en la medida en que se vuelven más como "nosotros" queremos que sean en lugar de como son ahora. De nuevo, se presenta una reproducción de los valores de la cultura dominante.

Otra contradicción tiene lugar cuando estas escuelas que sí llevan a cabo prácticas que se corresponden con un aprendizaje de calidad, son representadas como escuelas 'excluidas', separadas y jerárquicamente calificadas. En la línea de una escuela de calidad, la creatividad y el aprendizaje de esta escuela altamente marginada y estigmatizada es muy importante. Esta investigación lleva a pensar por qué ciertos barrios y sus habitantes son 
etiquetados como deficientes y cómo las divisiones de clases son cada vez más racializadas". (Beach, 2017). Su aprendizaje creativo y su forma de hacer contradicen las representaciones oficiales relativas a deficiencias de aprendizaje. Éstas demuestran un fuerte ingenio que contrasta con las perspectivas de déficit y demuestran que son capaces de aprender, que sus antecedentes no son un problema sino un valor en este aprendizaje. LLevan a cabo prácticas que reconocen las necesidades de todos los alumnos y lo hace toda la escuela. Realmente sólo necesitan un reconocimiento genuino de (y apoyo básico para) su capacidad y compromiso para hacerlo (Beach, 2017).

Se trata de una contradicción de la política en la práctica. Aunque la mayoría de las legislaciones declaran oficialmente la inclusión y el valor igual de todas las personas como estándares de justicia en la educación, podemos ver lo contrario. Se mantienen la marginación y la reproducción como prejuicios profundamente arraigados e históricamente empleados, sea por sexos, capacidades, clases y razas (Beach, 2017). Esto se mantiene en este nuevo contexto global de mercado y con la elección de centro escolar que apoya el estado capitalista, formando un complejo de instituciones que actuan como intereses de clase y donde la justicia social y la equidad no es posible. Las representaciones sobre la discapacidad, los antecedentes étnicos, la ubicación del hogar o la clase social siguen siendo referentes para el tratamiento diferenciado y para los resultados en la educación. En ese sentido, aunque la educación se expresa formalmente en la política oficial desde la neutralidad académica, sigue siendo, tanto en teoría como en práctica, culturalmente sesgada y social y culturalmente reproductiva(Beach, 2017).

Las experiencias de esta escuela con población en situación de pobreza en un barrio periurbano muestran cómo las características de estos espacios no son tomados en cuenta adecuadamente en los análisis habituales del problema de la educación en estas escuelas. Beach et al., (2013) señalan que la segregación social se aborda a través de prácticas bien intencionadas que reproducen y no desafían el orden social dominante, contribuyendo a que la población, de forma natural, vivan como ciudadanos de segunda clase (Beach \& Sernhede, 2012). Los bajos resultados de algunas escuelas parecen reforzarse por políticas de segregación y discriminación, que no son causadas principalmente por maestros o por las escuelas (Beach \& Dovemark, 2009; Möller, 2010).

Es importante señalar que las escuelas pueden aprender y sus profesores no se sienten desinteresados o indiferentes, y las familias están involucradas en sus escuelas y en el aprendizaje. Esto significa que no es la cultura y el trasfondo de los alumnos el problema, sino los valores de la herencia cultural de la clase media, la historia de la dominación de esta clase en la política del sector público y la auto-asumida superioridad de su código educativo en las principales organizaciones educativas, políticas y planes de estudios_(Beach, 2017).

\section{Observaciones finales}

Hay varias implicaciones en el artículo sobre el desafío actual de la educación para grupos de población que se encuentran en situación de desventaja. Una de ellas se refiere a una política de diferencia que debe ser desestimada y que sin embargo gana poder a través de la representación y categorización de las escuelas y los grupos de población a partir de supuestos de la superioridad de otros grupos y escuela, tan arraigados en nuestras estructuras políticas, institucionales y legales. De otra parte, parece que rara vez se toma plenamente en cuenta en las decisiones políticas o los informes políticos y mediáticos lo que influye en los resultados educativos de las escuelas que se encuentra en situación de desventaja.

\section{Referencias}

Ainscow, M., Booth, T. \& Dyson, A. (2006). Improving schools, developing inclusion. New York: Routledge.

Apple, M. W. (1996). Política cultural y educación. Madrid: Morata.

Arnaiz, P. (2003). Educación inclusiva: una escuela para todos. Málaga: Aljibe.

Ball, S. (1994). Education reform: A critical and post-structural approach. McGraw-Hill Education.

Barnard, W. M. (2004). Parent involvement in elementary school and educational attainment. Children and youth services review, 26(1), 39-62.

Beach, D. (2010). Neoliberal Restructuring in Education and Health Professions in Europe Questions of Global Class and Gender. Current Sociology, 58(4), 551-569.

Beach, D. (2017). Whose justice is this! Capitalism, class and education justice and inclusion in the Nordic countries: race, space and class history. Educational Review. http://www.tandfonline.com/doi/full/10.1080/00131911.2017.1288609

Beach, D., \& Dovemark, M. (2009). Making 'right'choices? An ethnographic account of creativity, performativity and personalised learning policy, concepts and practices. Oxford Review of Education, 35(6), 689-704.

Beach, D., Dovemark, M., Schwartz, A. \& Öhrn, E. (2013). Complexities and contradictions of educational inclusion-A meta-ethnographic analysis. Nordic Studies in Education, 33(4), 254-268.

Beach, D., \& Sernhede, O. (2012). Learning processes and social mobilization in a Swedish metropolitan hip-hop collective. Urban Education, 47(5), 939-958.

Bernal, J.L (2005). Parental choice, social class and market forces: the consequences of privatization of public services in education. Journal of Education Policy, 20(6), 779-792.

Bergnehr, D. (2015). Advancing home-school relations through parent support? Ethnography and Education, 10(2), 170-184.

Bolívar, A. (2006). Familia y escuela: dos mundos llamados a trabajar en común. Revista de educación, 339, 119-146.

Bouakaz, L. (2007). Parental involvement in school: What promotes and what hinders parental involvement in an urban school. Malmö University, Lärarutbildningen.

Boylan, M., \& Woolsey, I. (2015). Teacher education for social justice: Mapping identity spaces. Teaching and teacher education, 46, 62-71.

Calderón, I. (2014). Sin suerte pero guerrero hasta la muerte: pobreza y fracaso escolar en una historia de vida. Revista de Educación, 363, 184-209.

Carrasco, S., Pamiés, J., Bertran, M. (2009). Familias inmigrantes y escuela: Desencuentros, estrategias y capital social1. Revista Complutense de Educación, 20(1), 55-78.

Castro, M., Expósito, E., Lizasoain, L., López, E., y Navarro, E. (2014). Participación familiar y rendimiento académico. Una síntesis meta-analítica. En, Consejo Escolar del Estado (Ed.). La participación de las familias en la educación escolar, 87-109. Madrid: MECD.

Chusseau, N., \& Hellier, J. (2011). Educational systems, intergenerational mobility and social segmentation. The European Journal of Comparative Economics, 8(2), 203.

Cerletti, L. (2013). Enfoque etnográfico y formación docente: aportes para el trabajo de enseñanza. Pro-Posições 24, 2(71), 81-93.

Clavero, N. (2016). Ábreme con ilusión. Huesca: Editorial Pirineo.

Conteh, J., \& Kawashima, Y. (2008). Diversity in family involvement in children's learning in English primary schools: Culture, language and identity. English Teaching, 7(2), 113-125. 
Crozier, G. \& Davies, J. (2007). Hard to reach parents or hard to reach schools? A discussion of home-school relations, with particular reference to Bangladeshi and Pakistani parents. British Educational Research Journal 33(3), 295-313.

Delamont, S. y Atkinson, P. (1994). Fighting Familiarity. New Jersey: Hampton Press.

Eisenhart, M. (2016). A matter of scale: multi-scale ethnographic research on education in the United States. Ethnography and Education, 12(2), 134-147.

Engelbrecht, P (2013). Teacher education for inclusion, international perspectives. European Journal of Special Needs Education, 28(2), 115-118.

Epstein, J. L. (2001). School, Family and Community partnerships. Preparing educators and improving schools. Boulder CO: Westview Press.

Escardíbul, J. O., \& Villarroya, A. (2009). The inequalities in school choice in Spain in accordance to PISA data. Journal of Education Policy, 24(6), 673-696.

Escudero, J. M. y Martínez, B. (2012). Las políticas de lucha contra el fracaso escolar: ¿programas especiales o cambios profundos del sistema y la educación? Revista de Educación, Ext., 174-193.

Essomba, M.A. (2012) Inmigración, sociedad y educación en la UE. Hacia una política educativa de plena inclusión. Cultura y Educación, 24(2), 137-148.

Fan, X. \& Chen, M. (2001). Parental involvement and students' academic achievement: A meta-analysis. Educational psychology review, 13(1), 1-22.

Flores, R., Gómez , M. y Renes, V. (2016). La transmisión intergeneracional de la pobreza: factores, procesos y propuestas para la intervención. Madrid: Fundación FOESSA

Garreta, J. (2014). La participación de las familias en la escuela: una cuestión pendiente. Documentación Social. Revista de Estudios Sociales y Sociología Aplicada, 171, 101-124.

Gewirtz, S., Ball, S. J., \& Bowe, R. (1995). Markets, choice, and equity in education. Maidenhead: Open University Press.

Gil, L. R. (1966). Barrio de la parroquia de Nuestra Señora del Perpetuo Socorro. Argensola. Revista de Ciencias Sociales del Instituto de Estudios Altoaragoneses, 61, 65-79.

Gil, M., De Pablos, L., Martínez, M. (2010) Los determinantes socioeconómicos de la demanda de educación superior en España y la movilidad educativa intergeneracional. Hacienda Pública Españolal Revista de Economía Pública, 193, 75-108.

Goodson, I. F., \& Lindblad, S. (Eds.). (2011). Professional knowledge and educational restructuring in Europe (4). Rotterdam: Sense Publishers.

Gudmundsson, G. (2013). Excluded youth in itself and for itself-Young people from immigrant families in Scandinavia, In, G. Gudmundsson, D. Beach, V. Vestel (ed.), Young people and marginalisation: Young people from immigrant families in Scandinavia, 1-9. London: Tufnell Press

Hale, A., Snow-Gerono, J., \& Morales, F. (2008). Transformative education for culturally diverse learners through narrative and ethnography. Teaching and Teacher Education, 24(6), 14131425.

Harris, A., \& Goodall, J. (2008). Do parents know they matter? Engaging all parents in learning. Educational Research, 50(3), 277-289.

Jackson, M., Jonsson, J. O., \& Rudolphi, F. (2012). Ethnic inequality in choice-driven education systems: A longitudinal study of performance and choice in England and Sweden. Sociology of Education, 85(2), 158-178.

Jacobs, A. (2008). Perspectives on parent involvement: how elementary teachers use relationships with parents to improve their practice (Tesis Doctoral). University of Maryland, College Park.
Lareau, A. (2000). Home advantage. Social class and parental intervention in elementary education. Lanham, MD: Rowman \& Littlefield Publishers.

Lewis, L., Kim, Y. \& Bey, J. (2011). Teaching practices and strategies to involve inner-city parents at home and in the school. Teaching and Teacher Education, 27(1), 221-234.

Lindsay, G. (2009). Parent support advisor pilot evaluation. Final Report. London: DCSF.

Logan, J. R., Minca, E., \& Adar, S. (2012). The geography of inequality: Why separate means unequal in American public schools. Sociology of education, 85(3), 287-301.

López-Melero, M. (2012). La escuela inclusiva: una oportunidad para humanizarnos. Revista Interuniversitaria de formación del profesorado, (74), 131-160.

Martínez, B. (2011). Luces y sombras de las medidas de atención a la diversidad en el camino de la inclusión educativa.Revista Interuniversitaria de Formación del profesorado, (70), 165-184.

Mills, C. \& Gale, T. (2004). Parent participation in disadvantaged schools: Moving beyond attributions of blame. Australian Journal of Education, 48(3), 268-281.

Möller, A. (2013). Cultural racism in liberal democratic education in Sweden, In, G. Gudmundsson, D. Beach, V. Vestel (ed.), Young people and marginalisation: Young people from immigrant families in Scandinavia, 133-154. London: Tufnell Press

Moreno, F. J. M., y Bruquetas, M. (2011). Inmigración y Estado de bienestar en España. Barcelona: Obra Social, Fundación la Caixa.

Muñoz, M.; Vázquez, C. y Vázquez, J.J. (2003). Los límites de la exclusión. Madrid: Témpora.Caja Madrid.

Niemeyer, B. (2006). El aprendizaje situado: una oportunidad para escapar del enfoque del déficit. Revista de Educación, 341, 99-122.

Öhrn, E. (2012). Urban education and segregation: the responses from young people. European Educational Research Journal, 11(1), 45-57.

Olmedo, A. (2008). De la Participación Democrática a la Elección de Centro: las Bases del Cuasimercado en la Legislación Educativa Española. Education Policy Analysis Archives, 6(21), 1-35.

San Fabián, J.L. (1996). El centro escolar y la comunidad educativa ¿un juego de metáforas? Revista de Educación 309, 195-215.

Schecter, S. R. \& Sherri, D. L. (2009). Value added? Teachers' investments in and orientations toward parent involvement in education. Urban Education, 44(1), 59-87.

Slee, R. (2012). La escuela extraordinaria: exclusion, escolarización y educación inclusiva. Madrid: Morata.

Smith, J. G. (2006). Parental involvement in education among low-income families: A case study. School Community Journal, 16(1), 43.

Susinos, T y Parrilla, A. (2008). Dar la Voz en la Investigación Inclusiva. Debates sobre inclusión y exclusión desde un enfoque biográfico-narrativo. REICE Revista electrónica iberoamericana sobre calidad, eficacia y cambio en educación, 6(2), 157-171.

Theodorou, E. (2008). Just how involved is 'involved'? Re-thinking parental involvement through exploring teachers' perceptions of immigrant families' school involvement in Cyprus. Ethnography and Education, 3(3), 253-269.

Vigo, B., Dieste, B., y Julve, C. (2016). Voces sobre participación de las familias en la escuela y éxito escolar. RASE: Revista de la Asociación de Sociología de la Educación, 9(3), 320-333.

Vigo, B., Dieste, B., y Thurston, A. (2016). Aportaciones de un estudio etnográfico sobre la participación de las familias a la formación crítica del profesorado en una escuela inclusiva. Revista Electrónica Interuniversitaria de Formación del Profesorado, 19(1), 1-14. 
Vigo, B., \& Soriano, J. (2014). Teaching practices and teachers' perceptions of group creative practices in inclusive rural schools. Ethnography and Education, 9(3), 253-269.

Vigo, B. \& Soriano, J. (2015). Family involvement in creative teaching practices for all in small rural schools. Ethnography and Education, 10(3), 325-339.
Wenger, E. (2001). Comunidades de práctica: aprendizaje, significado $e$ identidad. Barcelona: Paidós.

Woods, P. (1996). Researching the art of teaching. Ethnography for educational use. London: RoutledgeFalmer. 